\title{
Necesidades de información de los padres para su participación en el proceso de parto* $^{*}$
}

\author{
Information Needs of Fathers for their Participation in Childbirth \\ Necessidades de informação dos pais para sua participação no processo de parto
}

Yury Marcela Arenas Cárdenas a

DOI: https://doi.org/10.11144/Javeriana.ie22.nipp

Universidad de Boyacá, Colombia

yumarcelis@gmail.com

ORCID: https://orcid.org/0000-0003-0736-706X

Recibido: 10 Marzo 2020

Aceptado: 15 Julio 2020

Publicado: 30 Diciembre 2020

\section{Resumen:}

Introducción: la participación del padre en el trabajo de parto le abarca una serie de decisiones para actuar libremente según su experiencia durante el nacimiento. Así, la presencia y preparación de los padres durante el evento se hace necesaria para el bienestar de la diada; por tal motivo, la investigación tiene el objetivo de ampliar conceptualmente las necesidades de información que requieren los padres para su participación en el proceso de parto. Materiales y Métodos: estudio cualitativo descriptivo interpretativo bajo la técnica de análisis de contenido dirigido. Se realizaron entrevistas de profundidad a 30 participantes. En el proceso de análisis, codificación y categorización de la información realizaron con apoyo de Atlas Ti. Versión 6.2. Resultados y Conclusiones: los resultados ampliaron conceptualmente las necesidades de información de los padres, los cuales se describieron y agruparon en tres categorías establecidas previamente por la literatura: Información como necesidad de acompañamiento, información como necesidad de expresar sentimientos, entrenador "entrenado" para el parto. Los padres manifiestan varios sentimientos que generalmente se intensifican con el progreso del trabajo de parto, este estudio evidencia que entre ellos la incertidumbre y el temor, son eventualmente ocasionados por la falta de información y el desconocimiento. El significado a la información se relaciona directamente con el papel que pueden desempeñar dentro de la sala de parto, la cual les permite interpretar y comprender el proceso que atraviesa la mujer, por consiguiente, desenvolverse con confianza mientras se da el nacimiento.

Palabras clave: Conducta paterna, parto obstétrico, salas de parto, información, educación.

\section{Abstract:}

Introduction: The participation of the father in labor encompasses a series of decisions to act freely according to their experience during childbirth. Thus, the presence and preparation of fathers is needed during the event for the wellbeing of the couple. For this reason, the objective of the research was the conceptual expansion of the information needs required by fathers for their participation during childbirth. Materials and Methods: interpretive descriptive qualitative study under the targeted content analysis technique. In-depth interviews were carried out with 30 participants. Atlas Ti. Version 6.2 was used for the analysis, codification, and categorization of the information. Results and Conclusions: the results expanded conceptually upon the information needs of the fathers. These needs were described and grouped in three categories previously established in literature: information as need for support, information as the need to express feelings, and trainer "trained" for childbirth. Fathers display several feelings that are generally intensified as the labor progresses; this study shows how uncertainty and fear are eventually caused by ignorance and a lack of information. The meaning of information is directly related to the role they can play in the delivery room, which allows them to better understand the process the woman is going through. Consequently, this allows them to act confidently as the birth is happening.

Keywords: Paternal behavior, obstetric birth, delivery rooms, information, education.

\section{Resumo:}

Introdução: a participação do pai no trabalho de parto abrange uma série de decisões para agir livremente de acordo com a experiência durante o nascimento. Assim, a presença e preparação do pai durante o evento é necessária para o bem-estar da díade; por tal motivo, a pesquisa objetiva ampliar conceitualmente as necessidades de informação requeridas pelos pais para sua participação no processo de parto. Materiais e Métodos: estudo qualitativo descritivo interpretativo sob a técnica de análise de conteúdo dirigido. Realizaram-se entrevistas a profundidade a 30 participantes. O processo de análise, codificação e categorização da informação foi

Notas de autor

\footnotetext{
a Autora de correspondencia. Correo electrónico: yumarcelis@gmail.com
} 
realizado com suporte de Atlas Ti. Versão 6.2. Resultados e Conclusões: os resultados ampliaram conceitualmente as necessidades de informação dos pais, as quais foram descritas e agrupadas em três categorias estabelecidas previamente pela literatura: Informação como necessidade de acompanhamento, informação como necessidade de expressar sentimentos, treinador "treinado" para o parto. Os pais manifestam vários sentimentos que geralmente intensificam-se com o avanço do trabalho de parto; este estudo mostra que entre eles a incerteza e o medo são ocasionados pela falta de informação e pelo desconhecimento. O significado das informações é relacionado diretamente com o papel que podem desempenhar dentro da sala de parto o que lhes permite interpretar e compreender o processo pelo qual a mulher passa e, portanto, agir com segurança enquanto o parto ocorre.

Palavras-chave: Comportamento paterno, parto obstétrico, salas de parto, informação, educação.

\section{Introducción}

La presencia del padre en el parto, sus acciones y decisiones, determinan una serie de eventos que se relacionan estrechamente con su propia experiencia del nacimiento, a su vez, esta experiencia se relaciona con la información y los conocimientos que posee el padre del evento (1). Si bien, la contribución de los padres durante el proceso gestacional es fundamental para comenzar a crear el vínculo con sus hijos $(2,3)$; lo es también en instante del nacimiento en el que se convierten en personas importantes para el bienestar de la diada (4).

Markowska et al., en su estudio realizado a 250 parejas que participaron en partos naturales, basado en la aplicación de una encuesta prospectiva, reportan que las mujeres sienten mayor satisfacción con compañía, destacando la presencia de la pareja, significaba apoyo físico y psicológico (5). La Organización Mundial de la Salud (OMS), refiere en sus recomendaciones del 2015 que, en las mujeres que tuvieron compañía con apoyo, hubo una leve reducción en intervenciones en el parto (6).

La percepción positiva de los padres en la experiencia del nacimiento, se genera cuando se les permite interactuar, realizar preguntas, obtener información y apoyo del personal de salud encargado (4, 7). No obstante, tienden a experimentar sentimientos abrumadores: temor, desolación, exclusión, angustia e incluso síndrome postraumático si se les permite ingresar sin preparación previa, o sin recibir la información adecuada para hacerlo $(4,8,9,10,11,12)$.

El sentir de los padres durante el proceso del nacimiento, tiende a ser frustrante cuando no se sienten apoyados, incluidos e informados en este contexto $(4,7,12)$. Esta es la razón que fundamenta el propósito del estudio, al profundizar en las necesidades de información que requieren los padres para su participación en el trabajo de parto.

Los resultados del presente estudio proporcionan instrumentos al profesional de Enfermería para plantear cuidados intervencionistas a las mujeres en trabajo de parto vinculando al padre en el nacimiento a través de una comunicación efectiva e interactiva. Así mismo, aboga hacia la promoción de políticas sociales e institucionales que propendan a fortalecer los derechos del hombre como padre, frente a la equidad de género y el descubrimiento de las nuevas masculinidades dentro del proceso de gestación y parto.

\section{Materiales y métodos}

Se realizó un estudio cualitativo descriptivo interpretativo bajo la técnica de análisis de contenido dirigido, con la finalidad de documentar y ampliar conceptualmente las necesidades de información que evidencian los padres durante su experiencia en el parto y la información significativa para su participación en el proceso (13).

Se adquirió la información a través de entrevistas personalizadas semiestructuradas, grabadas en audio y realizadas a partir de un muestreo intencional hasta lograr el desarrollo de las propiedades de las categorías en sus rangos más amplios y posibles de datos; determinándose la saturación teórica con 30 padres que 
cumplieron criterios de selección cómo: presenciar el trabajo de parto de su pareja por primera vez, cuyas parejas tuvieron un parto eutócico y un nacimiento exitoso y que fueran atendidos en la sala de partos del Hospital San Rafael de Tunja durante el primer semestre del año 2018. Se llevaron notas de campo posterior a cada entrevista. Las características sociodemográficas de los participantes durante el estudio fueron de 16 de origen rural, y 14 urbano; 19 eran padres por primera vez, 8 tenían su segundo hijo y 3 tenían su tercer y cuarto hijo; 13 estaban casados y 17 vivían en unión libre; con edades entre los 18 a los 46 años.

A través del proceso de análisis de la literatura consultada, se establecieron tres categorías que posteriormente fueron detalladas a partir de la codificación y su descripción minuciosa. Para el análisis y codificación de los datos se sirvió de la herramienta Atlas Ti versión 6.2.

De acuerdo con los criterios éticos, se actuó bajo la resolución 008430, donde se establecen las normas técnicas, científicas y administrativas para la investigación en salud en Colombia y los lineamientos éticos contemplados en el Código de Núremberg y el código de Helsinki (14).

Una vez informadas las características del proyecto, los participantes del estudio tomaron la decisión libre de participar; se realizó la firma del consentimiento informado y se dirigieron hacia el lugar de la entrevista según la comodidad del participante, la mayoría de las entrevistas se realizaron en las instalaciones del hospital o cerca de éste, teniendo en cuenta que se abordó a los padres en un periodo no mayor a 24 horas posterior al nacimiento de sus hijos. Se establecieron criterios de validez por triangulación y saturación de los datos, la credibilidad por el reconocimiento de los resultados por 10 participantes del estudio, la transferibilidad con la descripción exhaustiva de los datos del estudio y sus participantes, la confirmación y relevancia desde la criticidad de otro investigador. Las entrevistas transcritas textualmente, y fueron identificadas con la inicial "P", seguida de un código numérico de acuerdo con el orden cronológico de la entrevista, con la finalidad de garantizar la privacidad y la intimidad de los padres.

\section{Resultados}

Inicialmente se establecieron de la literatura tres categorías: información como necesidad de acompañamiento, información como necesidad de expresar sentimientos a lo desconocido y entrenador "entrenado" para el parto. A continuación, se describen cada una de ellas en función de su ampliación conceptual a la literatura y la subcategorización establecida.

\section{Información como necesidad de acompañamiento}

Es expresada por los padres como la definición y obtención de información durante el proceso de parto, las personas de quienes la reciben, el canal por el que se transmite y el momento oportuno de recibirla. Establecida en las siguientes subcategorías:

\section{- Dando un significado a la información}

Comprender el evento del parto para los padres se determina con el reconocimiento de la situación y el entorno en el que se desenvuelven. Reconocen distanciamiento y desvinculación del proceso por su condición de hombres, lo que disminuye la posibilidad de informarse, es así, que presenciarlo genera un amplio conocimiento.

La información que esperan recibir los padres debe basarse en las acciones que se realizan a su pareja y las eventualidades que pueden presentarse. De igual forma, desean información sobre equipos (monitores fetales, monitores de signos vitales, bombas de infusión), procedimientos y acciones (amniotomía, medicalización, 
tactos, etc.), que sea clara y concisa de tal forma que los padres puedan relacionarlos con la evolución del nacimiento y el posible beneficio que conllevan en la mujer y su hijo por nacer:

Emmm... como bueno porque uno se entera de cosas que uno ni idea, que uno de hombre como que la deja como que pasar, uno como que espera que ella salga y ya, como que uno espera, pero uno aprende y valora todo eso. (1:381-1:623. P3) ${ }^{1}$

Sí, bueno digamos que no con cierta profundidad, pero digamos que lo básico ęsí?, esto es, esto es para esto, esto sirve para esto... y todo en beneficio de su esposa y su hijo... considero que es importante la información. (2:2838-3:239. P2)

Así mismo, la información y preparación previa al parto, es fundamental para los padres, ya que determina las acciones que pueden desempeñar dentro de la sala y en función de su pareja, evitando sentirse en un estado manifestado por ellos como de "shock o robot". Así mismo, consideran que debe ser en un tiempo cercano a la fecha probable de parto establecida desde el inicio de la gestación, personalizada idealmente, contemplando también medios impresos y audiovisuales, incluyendo conferencias y talleres específicos para padres, en horarios flexibles a sus actividades laborales y con un tiempo aproximado de 60 minutos, y que se les permita tomar la decisión de ingresar o no al parto.

Y... pues claro, en ese momento uno queda... superrr... quede en shock porque... yo la verdad no había... no lo había planeado... y tampoco no había pensado entrar... como no sabe qué hacer, no sabe si lo que hasta haciendo está haciendo bien o está haciendo mal... porque la verdad... quede como un robot (riendo). (1:2246-1:2696. P30)

Puesss en mi caso ya como vengo del pueblo, fuera sido en el pueblo si, unos ocho días antes, que le fueran... fueran, como una lección, si esto va a pasar allá, si se atreven a entrar o vamos a estar sometidos a esto y esto. (3:1795-3:2073. P15)

Ante la falta de información los padres evidencian incertidumbre, desorientación e incluso frustración, debido al desconocimiento y actúan según lo indica el personal a sus parejas, pero no a ellos directamente; no sienten propiedad en su actuar, manifiestan pensamientos negativos ante las acciones realizadas por el personal (administración de medicamentos, episiorrafia, toma de signos, instrumentación del parto) y los cambios fisiológicos en la mujer (ruptura de membranas, llanto, mala respiración, expulsión fetal, alumbramiento). El presenciar el evento se convierte en un suceso sorpresivo, estresante y temido para los padres, impidiendo comunicarse con los profesionales de la salud, relacionado a la falta de confianza y la creencia de ocasionar inconvenientes o distractores que afecte la atención hacia sus parejas e hijos. La mala actitud y el mal humor de algunos profesionales, dificultó también el acercamiento. Conductas y pensamientos que tendieron a cambiar en los padres cuando eran informados: "hubo un momento donde la cabecita de la niña era... es una niña, ya estaba por fuera, y entonces dejo de pujar, entonces yo vi como que la niña se puso negrita, moradita" (1:1501-1:1832. P15). "Sí me acerqué, pero uno, uno... uno arriesga a los doctores que de pronto los interrumpe y... entonces eso no se hace" (5:145-5:262. P10).

... Porque hay casos en los que por ejemplo... las enfermeras son bravas, y a lo que no esto... porque igual que culpa uno, a veces hace una pregunta y no es porqueee no la quiera hacer, sino que es que uno tiene una duda. (3:2760-3:3173. P26)

Poseer algún tipo de información externa sobre el proceso del parto, ya sea por familiares, videos o programas informativos, brindan tranquilidad en los padres, que aseguraron tener una idea del evento, no obstante, es la propia experiencia la que genera mayor confianza y orgullo en sí mismos:

pues es que lo que pasa es que yo el otro días... vi unaaa... vi un parto en televisión, en Discovery, y como que me metí en el tema, sí me entiende, o sea me profundicé mucho en el tema, dije haaa listo, o sea me voy a, a alistar psicológicamente... como si lo estuvieraaa viviendo yo y así lo fue, o sea... esatitico, casi lo mismo. (3:1712-3:2252. P26)

\section{- Comprender el proceso de mi pareja}

Identificar, comprender y asumir el acompañamiento dentro del proceso de parto, surge a través de la observación del entorno y la escucha al personal de salud. El proceso fisiológico de la mujer, las contracciones, el dolor y el parto como tal, producen impactos positivos o negativos para los padres, estos ligados a la 
información o desinformación frente al evento. Lo que puede llevarlos a buscar fuentes de información no confiables:

enriquecedor porque... digamos que supe, sí, me di cuenta así de primera mano emmmm lo que sufre ¿sí? La persona que tiene el bebé, en este caso mi esposa... no es un trabajo fácil, no es un trabajo corto... es muy complejo ¿sí? ... uno sabe que tiene un compromiso con la familia, y digamos queee ese, ese acto, en mi punto de vista reforzó mucho más ese compromiso que tengo con mi esposa y con mi hijo. (1:1315-1:1890. P2)

El dolor experimentado por la mujer en trabajo de parto genera abatimiento en los padres, revelan frustración al no obtenerle un alivio, ni un nacimiento más rápido. Pese a esto, los padres consideran su presencia fundamental, asumiendo un papel protector mientras brindan comprensión, afecto y apoyo a sus parejas:

[en la sala de espera] estaba pensando en mi esposa. O si estaba sufriendo, ooo... o que porque..., yo sabía que iba a... estaba sufriendo, entonces no sabía cómo estaría ella, si estaría desesperada, si estaría angustiada o si estaba algo calmada o concentrada en su trabajo de parto... no sabía cómo estaba, hasta que la vi [refleja tristeza en sus palabras, tono de voz bajo]. (11:11.P16)

\section{Acompañamiento profesional}

La labor del personal de salud es reconocida como valiosa, casi "heroica" otorgando confianza al padre; la cercanía, el ser tenidos en cuenta, el diálogo y la información, evidencia en los padres apoyo, satisfacción y sentimientos positivos frente a su participación; no obstante, se percibe malestar, emociones negativas, temor, ansiedad e incluso agresión por parte del personal de salud ante la desinformación, el desconocimiento protocolario y las conductas evasivas. "pues uno se siente como... no sé cómo... casi como... estar ahí con los doctores ahí al pie, como estar ayudando, como si fueran unos héroes ellos, entonces uno se siente así" (3:76-3:248. P10).

\section{Información como necesidad de expresar sentimientos a lo desconocido}

Involucra todas las manifestaciones emocionales que los padres guardaron o expresaron libremente durante el proceso de parto y el acompañamiento a sus parejas. Sustentado por las subcategorías:

\section{- Contener sentimientos}

Los padres se enfrentan a una variedad de encrucijadas emocionales durante el proceso de parto, por un lado, la enorme alegría que genera el hecho de convertirse en padres y por otro el temor, la angustia y la tristeza de observar a la pareja enfrentar el dolor del trabajo de parto sin remedio alguno, emociones que deben cohibirse, según ellos, por su misma condición de hombres (símbolo de fuerza) y porque pueden desencadenar situaciones negativas en la mujer y el desarrollo del parto.

Los padres asumen la experiencia como enriquecedora en varios aspectos: para ellos como padres, para el núcleo familiar y la relación en pareja, manifiestan valorar la fuerza "verraquera" de la mujer y su capacidad de aguantar el dolor para enfrentar la situación; incluso, reflexionan frente a su condición de hijos, padres y esposos, hacen del proceso de parto una experiencia valiosa en sus propias vidas:

mmm pues yo la vi siempre como ya, como cansada, como sin alientos, pues no demostrarle de que, que, que... estaba preocupado sino a darle ánimo, porque si me veía preocupado pues se iba a preocupar ella más. Entonces era como jugarle a la contraria de los sentimientos que tenía ella. (27:27. P16) 


\section{- Revelar sentimientos}

La expresión de sentimientos se refleja con acciones de apoyo, caricias, palabras y transmisión de fuerza. Durante el nacimiento y el contacto piel a piel, los padres en su mayoría expresaron con llanto sus emociones, considerando el suceso como la mejor experiencia de sus vidas: "lo primero que hice cuando nació mi bebé fue llorar, me considero una persona fuerte, pero ese momento de ver nacer a mi hijo, si me conmovió totalmente" (2:466-2:654. P2).

\section{Entrenador "entrenado" para el parto}

Ante la situación, los padres tienden a convertirse en "entrenadores" de sus parejas, asumiendo un papel orientador y protector, se involucran y aumentan su receptividad para entrenarse, para aprender del entorno, de lo que observan y escuchan.

\section{- Desempeñando un papel dentro del parto}

Las salas de parto se convierten en un ambiente de preparación inmediata ante la escasa o falta de información y conocimiento de los padres frente al evento, así el papel que desempeñan adquiere un gran significado con acciones concretas como conducir las respiraciones, masajes, palabras de aliento, miradas, entre otras, son consideradas, fundamentales por los padres al evidenciar cambios en la actitud de sus parejas:

inicialmente, creí queee era nada más la presencia ¿sí? digamos que presenciar el momento del nacimiento de mi hijo, pero después cuando iba pasando el tiempo durante el parto y el proceso de parto, me di cuenta de que es fundamental. (2:2078-2:2818. P2)

\section{- Conociendo el proceso del parto}

La participación y los conocimientos que los padres adquieren sobre la experiencia vivida evidencian confianza y orgullo, teniendo en cuenta que para ellos su presencia no es pertinente, por razones como el exceso de temor y el síncope. Los padres obtienen finalmente la capacidad y la seguridad de hablarle a otros padres sobre el proceso, aconsejar sobre las acciones, e incluso sugerirles la preparación previa que deben tener. Así mismo, se sienten privilegiados, advirtiendo su presencia en el evento como un derecho:

Importante si, que todos los papás ojalá puedan hacerlo, que vean esa etapa de la maternidad, porque pues, creo que esto nos da a valorar un poquito más las mujeres ¿no?, a saber cuál es su punto de entregarnos a nosotros los hijos, si, la mayoría de papas no valoramos ese punto sino siempre los vemos cuando ya están vestiditos, pero no contemplamos ese sufrimiento tan grande que tiene una madre. (3:512-3:610.P1)

\section{Discusión}

Las necesidades de información de los padres en el proceso del parto se evidencian durante su participación en el evento y a través, de su experiencia y el desempeño de su rol de padre. Los padres cursan inicialmente por una etapa de reconocimiento en la que limitan la expresión de sus emociones, sentimientos y percepciones; en el proceso, adquieren nuevos conocimientos que observan del entorno del evento; para finalmente apropiarse de la situación, enfrentarla y asumirla con pertenencia en la espera de un efecto positivo para su pareja y su hijo, descritos en este estudio. De tal forma que, durante el estudio, se evidencia con claridad que es la información 
la necesidad más manifiesta por los participantes, lo que se respalda por la literatura, en la que se enfatiza en la claridad y calidad de información que deben recibir los padres $(7,15)$.

En concordancia con otras investigaciones, la importancia de la información que reciben los padres, y la comunicación que pueden establecer con los profesionales de la salud, se considera un factor fundamental en el proceso, así, según Lewis et al., en su estudio realizado en Nepal, percibieron indispensable la comunicación entre los profesionales de la salud y los padres (11), aprovechando su presencia como apoyo a la mujer en proceso de parto $(7,15,16)$ y en donde se le brinde confianza y calidez también al padre (12), asistiendo sus acciones y decisiones en favor de su pareja y su hijo (15). Por el contrario, las experiencias negativas de participación del padre que se evidenciaron en este estudio, se relacionan con las barreras en la comunicación, la falta de acompañamiento profesional y el lenguaje técnico que comúnmente les resulta incomprensible y confuso; así mismo, ansiedad frente a proceso de parto difícil, lo que aumenta su preocupación y los pensamientos negativos frente al proceso de parto (10), incluso generando afecciones psicológicas, manifiestan que el apoyo y la información es un derecho que tienen como padres; sustentado en estudios como el de Darwin et al., en el que evaluaron la prevalencia de depresión y ansiedad en padres desde la concepción hasta el primer año de nacimiento (15).

Los sentimientos que relacionan a la vivencia del parto, están estrechamente relacionados con el temor y la preocupación, y se convierte en un tema complicado a tratar con los hombres, ya que consideran que su realidad masculina limita la expresión de emociones con libertad, por el contrario, estudios como el de Braide et al., revelan la resignificación de las identidades masculinas, y el nuevo rol que desempeña como cuidador e involucrándose en todas las actividades de la nueva familia (16); así mismo, otros estudios, sugieren que estos sentimientos negativos, pueden disminuir cuando el padre o el compañero masculino actúan como entrenador entrenado en el parto (17). Enfatizando la importancia que tiene la información y preparación del padre sobre el evento del parto y la necesidad de vivir una experiencia más positiva del nacimiento.

Finalmente es importante determinar que es Enfermería quien desarrolla un rol educativo basado en la comunicación con las personas y sustentado por teorías disciplinares; Peplau (18), Trabelvee (19), Meleis (20), evidenciando las necesidades de las personas en establecer procesos de comunicación a partir de sus situaciones particulares e individuales de salud o enfermedad, donde se guíen sus acciones a través de la retroalimentación y conduciéndolas a desarrollar respuestas constructivas y resolutivas, dándole sentido a sus experiencias $(18,19,20,21)$.

\section{Conclusiones}

Es la información, parte del acompañamiento que desean recibir los padres durante el proceso de parto, pero también, es evidente que en muchos aspectos ésta es confusa, escasa, o nula por lo que es difícil establecer un canal de comunicación efectivo entre el personal de salud y los padres. Razón por la cual, los sistemas de salud deben generar espacios exclusivos de preparación para ellos.

El significado cultural de ser padre y la redefinición de la masculinidad trasciende las creencias sociales a partir, de acciones como la participación activa de los hombres en el parto y el fortalecimiento de su rol en la familia.

\section{Referencias}

1. Bond MJ. Paternal Involvement in Pregnancy Outcomes. Am J Mens Health [Internet]. 2013;4(4):285-286. https ://doi.org/10.1177/1557988310384842

2. Vesga L, Ruiz C. Percepción que tienen las gestantes sobre el cuidado de enfermería en la atención prenatal. Investig Enferm Imagen Desarr [Interet]. 2019;21(1):1-21. https://doi.org/10.11144/Javeriana.ie21-1.ptgc 
3. Johansson M, Fenwick J, Premberg. A meta-synthesis of fathers' experiences of their partner's labour and the birth of their baby. Midwifery [Internet]. 2015;31(1):9-18. https://doi.org/10.1016/j.midw.2014.05.005

4. Suto M, Takehara K, Yamane Y, Ota E. Effects of prenatal childbirth education for partners of pregnant women on paternal postnatal mental health: a systematic review and meta-analysis protocol. Syst Rev [Internet]. 2016;5(1):21. https://doi.org/10.1186/s13643-016-0199-3

5. Markowska S, Porçba R, Krawczyk P, Skrzypulec V. The preferences of parturient women and the participation of the child's father during birth. Neuroendocrinol Lett [Internet]. 2015;36(5):447-451. Disponible en: http://w ww.ncbi.nlm.nih.gov/pubmed/26707045

6. Organización Mundial de la Salud. Recomendaciones de la OMS para la conducción del trabajo de parto. OMS [Internet]. 2015;11-43. Disponible en: http://www.who.int/topics/maternal_health/directrices_OMS_parto _es.pdf

7. Emelonye A, Pitkäaho T, Aregbesola A, Vehviläinen K. Women's perceptions of spousal relevance in childbirth pain relief in four Nigerian hospitals. Sex Reprod Healthc [Internet]. 2017;12:128-132. https://doi.org/10.1016/j. srhc.2017.04.004

8. Phuong T. Factors influencing intention of elective cesarean section among pregnant women in Quang Ngai province, Vietnam. Factors Influ Intent Elect cesarean Sect among pregnant women. Quang Ngai Prov Vietnam. 2015;38(4):137-151. Disponible en: https://he01.tcithaijo.org/index.php/nah/article/view/33599

9. Uribe C, Contreras A, Hoga L. Presencia activa del padre en el nacimiento integral: significados atribuidos por padres y madres a los roles paternos. Rev. Chil. Obstet. Ginecol. [Internet]. 2018;83(1):22-26. https://doi.org/10.406 7/s0717-75262018000100022

10. Conesa-Ferrer MB, Canteras-Jordana M, Ballesteros-Meseguer C, Carrillo-García C, Martínez-Roche ME. Comparative study analysing women's childbirth satisfaction and obstetric outcomes across two different models of maternity care. BMJ Open. 2016;6(8):e011362. https://doi.org/doi.org/10.1136/bmjopen-2016-011362.

11. Lewis S, Lee A, Simkhada P. The role of husbands in maternal health and safe childbirth in rural Nepal: A qualitative study. BMC Preg Childbirth [Internet]. 2015;15(1):1-10. https://doi.org/10.1186/s12884-015-0599-8

12. Meier ME, Avillaneda L. A Literature Review of Paternal Involvement in Prenatal Care. 2015;15(1):10. Disponible en: http://digitalcommons.library.tmc.edu/jfs/vol15/iss1/10

13. Cáceres P. Análisis Cualitativo De Contenido: Una alternativa metodológica alcanzable. Psicoperspectivas. 2003;2(1):53-82.

14. Ministerio de Salud y Protección Social. Resolución número 8430 de 1993. Bogota: Ministerio de Salud y Protección Social; 1993 oct 4.

15. Darwin Z, Galdas P, Hinchliff S, Littlewood E, McMillan D, McGowan L, et al. Fathers' views and experiences of their own mental health during pregnancy and the first postnatal year: a qualitative interview study of men participating in the UK Born and Bred in Yorkshire (BaBY) cohort. BMC Preg Childb [Internet]. 2017;17(45). https://doi.org/10.1186/s12884-017-1229-4.

16. Braide ASG, Brilhante AV, Arruda CN, Mendonça FAC, Caldas JMP, Nations MK, et al. Sou homem e pai sim! (Re)construindo a identidade masculina a partir da participação no parto. Rev Panam Salud Púb. 2018;42:e190. https://doi.org/10.26633/RPSP.2018.190

17. Baldwin S, Malone M, Sandall J, Bick D. A qualitative exploratory study of UK first-time fathers' experiences, mental health and wellbeing needs during their transition to fatherhood. BMJ Open. 2019 sep. 13;9(9):e030792. https://doi.org/10.1136/bmjopen-2019-030792

18. Baldwin S, Malone M, Sandall J, Bick D. Mental health and wellbeing during the transition to fatherhood: a systematic review of first time fathers' experiences. JBI Database System Rev Implement Rep. 2018 nov.;16(11):2118-2191. https://doi.org/10.11124/JBISRIR-2017-003773

19. Ballon-Aguilar YA, Escalante Y, Huerta MM. Relación enfermera-paciente según la teoría de hildegard peplau en el servicio de centro quirurgico de un hospital nacional. [tesis licenciatura]. [Lima]: Universidad Peruana Cayetano Heredia; 2016. 
20. Meleis A. Transitions Theory: Middle Range and Situation Specific Theories in Nursing. New York: Springer Publishing Company; 2010. Cap. 1; p. 11-38.

21. Galvis MA. Teorías y modelos de enfermería usados en la enfermería psiquiátrica. Rev Cuid [Internet]. 17 de julio de 2015 jul. 17;6(2):1108-1120. https://doi.org/10.15649/cuidarte.v6i2.172

\section{Notas}

* Artículo original de investigación

1 Combinaciones correspondientes a la cita textual del código establecido en la entrevista realizada al participante, generadas automáticamente el programa de Atlas TI en el análisis de las entrevistas realizadas.

\section{Licencia Creative Commons CC BY 4.0}

Cómo citar este artículo: Arenas-Cárdenas YM. Necesidades de información de los padres para su participación en el proceso de parto. Investig Enferm Imagen Desarr. 2020;22. https://doi.org/10.11144/J averiana.ie22.nipp 